\title{
Autotrasplante Dental Utilizando Simulación Virtual y un Prototipo de Modelo de Impresión 3D
}

\author{
Dental Autotransplantation Using Virtual Simulation and a Prototype 3D Printing Model
}

\author{
González-Quintanilla David ${ }^{1,2}$; Juan Pablo Zamorano1; Eduardo Mella1; \\ Nicole Pinto1; Jorge Brisso'; Nicole Rodriguez ${ }^{1,3}$ \& Maximiliano Casa H. ${ }^{1,4}$
}

GONZÁLEZ-QUINTANILLA D.; ZAMORANO, J. P.; MELLA, E.; PINTO, N.; BRISSO, J.; RODRÍGUEZ, N. \& CASA, H. M. Autotrasplante dental utilizando simulación virtual y un prototipo de modelo de impresión 3D. Int. J. Odontostomat., 15(1):271$277,2021$.

RESUMEN: La técnica de autotrasplante dental se ideó como opción terapéutica ante la pérdida o ausencia de dientes por traumatismos, caries, agenesias, exfoliación temprana, iatrogenias, u otros factores. Consiste en extraer un diente desde su posición original para implantarlo en un solo acto quirúrgico en una zona edéntula, sea ésta un alveolo postextracción o alveolo confeccionado quirúrgicamente. Un resultado exitoso, requiere una meticulosa selección de candidatos, para esto, se debe considerar la ausencia de patologías y condiciones de orden local o sistémico que dificulten una adecuada cicatrización de heridas y reparación tisular. Nuevas herramientas para facilitar éxito clínico, como la planificación y simulación virtual, junto con la impresión de prototipos en tres dimensiones (3D), permitirían optimizar posibles osteotomías y una implantación del diente donante de manera fácil y en tiempos quirúrgicos mínimos, favoreciendo la disminución de la complejidad quirúrgica, además de la reparación y regeneración de tejidos pulpares y periodontales. El objetivo de este artículo es reunir una serie de consideraciones para optimizar la técnica de autotrasplante dental.

PALABRAS CLAVE: autotrasplante dental, simulación virtual, impresión 3D.

\section{INTRODUCCIÓN}

El autotrasplante dental se define como el traslado de un diente de su alvéolo a un alvéolo postextracción o alvéolo confeccionado quirúrgicamente en un mismo paciente (Aparicio et al., 2008; Kang et al., 2013). Es una técnica que gozó de amplio predicamento en los siglos XVIII y XIX, si bien con éxito relativo, pero que la escuela escandinava protocolizó hacia los años 50 del pasado siglo, permitiendo obtener buenos resultados de forma predecible. Esta técnica se ideó como opción terapéutica ante la pérdida de dientes por traumatismos, caries o agenesias (Giannobile \& Lang, 2016).

Tras décadas de utilización de los implantes dentales como solución para sustituir dientes perdidos tempranamente en zonas de función y/o estética importantes, se ha puesto en evidencia los problemas que puede representar su instalación en pacientes en crecimiento o con potencial crecimiento residual del proceso alveolar, lo que hace necesario reevaluar la conservación de dientes y la utilización de técnicas clínicas clásicas en este tipo de pacientes, por lo general niños y jóvenes de primera, segunda e inclusive tercera década de vida (Giannobile \& Lang).

Uno de los objetivos principales de la odontología es preservar estructuras dentales con fines funcionales y estéticos, por esto, la opción del autotrasplante ha ido ganando terreno. A diferencia de los implantes dentales, en caso de que un trasplante dental tenga éxito, el resultado clínico sería similar a otro diente natural (Sugai et al., 2010), es de-

\footnotetext{
${ }^{1}$ Facultad de Ciencias de la Salud. Universidad Autónoma de Chile, Santiago, Chile.

${ }^{2}$ Instituto de Ciencias Biomédicas, Universidad Autónoma de Chile, Chile.

${ }^{3}$ Cirujano Maxilofacial, Hospital de Carabineros de Chile, Santiago, Chile.

${ }^{4}$ Endodoncista, Director Especialidad de Endodoncia, Universidad Autónoma de Chile, Chile.
} 
GONZÁLEZ-QUINTANILLA D.; ZAMORANO, J. P.; MELLA, E.; PINTO, N.; BRISSO, J.; RODRÍGUEZ, N. \& CASA, H. M. Autotrasplante dental utilizando simulación virtual y un prototipo de modelo de impresión 3D. Int. J. Odontostomat., 15(1):271-277, 2021.

cir, idealmente con vitalidad pulpar y adecuado estado periodontal, evaluado radiográficamente, como un espacio continuo alrededor de la raíz con ausencia de reabsorción de esta y la presencia de una lámina dura, con preservación de cresta alveolar y la adaptación funcional (Tsukiboshi et al., 2002; Gupta et al., 2015).

La aplicación clínica de un modelo de prototipos rápidos asistidos por computadora (CARP) para estos procedimientos, se introdujo por primera vez en 2001, utilizando la adquisición de imágenes en 3D de tomográficas computarizadas de haz cónico (CBCT), seguido de la fabricación con impresoras 3D de estos prototipos con materiales biocompatibles, biodegradables y esterilizables mediante radiación gamma y beta, como el ácido poliláctico (Gulín Arias, 2014; Lee et al., 2001; Liu et al., 2019; Li et al., 2020). Esta técnica permitió a los cirujanos simular el contorno del hueso receptor utilizando los prototipos CARP impresos en 3D de dientes donantes y huesos alveolares receptores en tamaño real, antes del procedimiento quirúrgico. El uso de prototipos o modelos CARP para el autotrasplante dental, tiene la ventaja de minimizar el tiempo extra alveolar y el trauma en el diente donante, lo que aumenta la tasa de éxito de la cirugía (Jang et al., 2016). Las tasas de supervivencia para la técnica de autotrasplante convencional que utiliza dientes con raíces maduras varían del $59 \%$ al $81 \%$ a los 4 años y del 59,6 \% a los 10 años (Mejàre et al., 2004). La simulación con modelos CARP en 3D, mejoran las tasas de supervivencia de los dientes con raíces maduras hasta el $88,1 \%$ y el $68,2 \%$ a los 3 y 12 años, respectivamente (Jang et al., 2016). Esta mejora, es más pronunciada cuando se usan dientes de donantes con raíces inmaduras (Jang et al., 2013).

\section{CONSIDERACIONES PARA LA INDICACIÓN DE TRATAMIENTO}

Para un resultado exitoso, el autotrasplante dental requiere una meticulosa selección de candidatos. Se debe considerar individuos con buena higiene oral, ausencia de patologías y condiciones de orden local y sistémico asociadas a los tejidos conectivos, discrasias sanguíneas, enfermedades metabólicas y/o endocrinas, entre otras, que a partir de la inmunología y mecanismos biológicos dificulten una adecuada cicatrización de heridas y reparación tisular (Alvarez et al., 2019). Además, la valoración terapeutica, conductual y motivacional del paciente por un equipo multidisciplinario, compuesto por endodoncista, ortodoncista, rehabilitador oral y cirujano, entre otros profesionales, sería recomendable (Nimcenko et al., 2013).
El autotrasplante estaría indicado en situaciones como: agenesia, caries profunda que impide que el órgano dental sea restaurado, exfoliación prematura, malformación, fractura dental complicada que impida su restauración, iatrogenia que indique la extracción, fracaso en el tratamiento endodóntico, tratamiento protésico no viable u otra condición o factor que no permita su restauración o rehabilitación (Barrientos et al., 2012). Lo anterior, sujeto a la existencia de un diente sano que reúna características clínicas y radiográficas para ser trasplantado.

El sitio receptor deben examinarse con el máximo cuidado para garantizar la adaptabilidad del diente donante (Lee et al., 2001. Se debe solicitar idealmente tomografía computada o de haz cónico con reconstrucción 3D para análisis de grosor óseo, morfología de la zona y raíz del diente a extraer y a trasplantar para una adecuada evaluación y planificación del tratamiento en caso de que se lleve a cabo (Hamilton et al., 2012; Oh et al., 2018). Se requiere un soporte óseo con suficiente tejido queratinizado bien insertado, libre de infección y/o inflamación, para permitir la estabilización del diente en el sitio receptor, el que debe tener un tamaño acorde al diente a trasplantar (Oh et al., 2005; Clokie et al., 2014). El diente donante, idealmente debe tener anatomía radicular de fácil instrumentación en caso de futuras complicaciones con resolución endodóntica (Ahmed Asif et al., 2017) e idealmente ápice abierto con a lo menos 1,5 $\mathrm{mm}$ de diámetro para quecapilares sanguíneos invadan el canal radicular permitiendouna adecuada revascularización (Skoglund et al., 1981; Andreasen et al., 1990; Herrera et al., 2006).

En caso de enfermedad periodontal generalizada no controlada, dientes que pueden ser rehabilitados, índices de caries elevados o cuando el diente a suplantar no puede ser extraído por completo, no está indicado el autotrasplante (Barrientos et al.). También, se ha descrito que el ancho inadecuado del hueso buco-lingual - la preparación quirúrgica excesiva pueden provocar la reabsorción del reborde alveolar y la pérdida de la cobertura del hueso bucal, lo que puede afectar en gran medida la curación periodontal. Lo anterior y la falta de pared ósea bucal sería un predictor significativo de fracaso del autotrasplante dental (Mejàre et al.).

\section{PROTOTIPO DE SIMULACIÓN VIRTUAL E IMPRE- SIÓN 3D}

La planificación del tratamiento virtual del autotrasplante se ha informado en estudios previos 
GONZÁLEZ-QUINTANILLA D.; ZAMORANO, J. P.; MELLA, E.; PINTO, N.; BRISSO, J.; RODRÍGUEZ, N. \& CASA, H. M. Autotrasplante dental utilizando simulación virtual y un prototipo de modelo de impresión 3D. Int. J. Odontostomat., 15(1):271-277, 2021.

(Strbac et al., 2016; Verweij et al., 2017), que utilizaron software para cirugía guiada de implantes, así como la aplicación combinada de un modelo CARP e impresión 3D y un software de diseño asistido por computadora $(C A D)$ que generalmente se utiliza para prótesis fija (Oh et al., 2018).

Ha habido muchos informes sobre las ventajas de los modelo CARP para un diente donante en autotrasplante (Lee et al., 2001, 2012; Jang et al., 2013; Verweij et al., 2016). Estos promueven la construcción virtual de una cavidad de extracción provisional utilizando imágenes CBCT antes de la extracción del diente comprometido, además, de la obtención de la forma del diente donante y el hueso alveolar para su duplicación en los prototipos o modelos CARP mediante impresión 3D con materiales de naturaleza descrita previamente en este trabajo,de alta precisión, con margen de error promedio entre el diente real y el modelo CARP de solo 0,291 mm (Lee et al., 2012).

La morfología de la raíz de los dientes podría ser interpretada de manera diferente a la otorgada por la imagen CBCT. Para solucionar esta posible complicación, se podrían utilizar un escáner óptico 3D tipo (Cerec Omnicam) y un software CAD, utilizados comúnmente para restauraciones y prótesis fijas indirectas. El primero permitiría escanear los modelos en $3 \mathrm{D}$ de la réplica del diente donante y alvéolo receptor, sobre la base de su capacidad de medir objetos en 3 dimensiones aplicando un método de triangulación activa con una precisión clínicamente aceptable (Kurz et al., 2015). El programa CAD podría informar a través de la articulación de estos elementos de acuerdo con una posición de relación céntrica previamente establecida, el área que se prepararía en el alvéolo receptor mediante la presentación de un área de contacto prematura después de que la réplica del diente donante entre en contacto el alvéolo de extracción sin osteotomía (Oh et al., 2018).

La planificación, simulación y pruebas de adaptabilidad permitirían el autotrasplante del diente donante con un tiempo extraalveolar mínimo, sin almacenamiento y con ausencia de traumatismos (Jang et al., 2016; Verweij et al., 2016; Kafourou et al., 2017). Lo anterior, contribuye a la regeneración de la pulpa mediante la preservación de la vaina radicular epitelial de Hertwig, así como la de las células de ligamento periodontal a la reconstrucción del tejido conectivo y el hueso, proporcionando una revascularización rápida a la superficie de la raíz del diente donante, además de extracción innecesaria del hueso receptor (Lin et al., 1994; Arima et al., 2019).

\section{CONSIDERACIONES PREQUIRÚRGICAS}

Se debe considerar en primera instancia, el uso de técnicas de adaptación conductual para reducir los niveles de ansiedad fisiológica de los pacientes sean o no pediátricos durante el tratamiento (Al Halabi et al., 2019).

La utilización de enjuagues antisépticos previo a la atención odontológica favorecería la disminución de virus y bacterias en la cavidad oral. La elección del antiséptico debe ser basada en permitir una adecuada disminución de la carga bacteriana y viral salival, sin generar daño en la mucosa oral (Meng et al.,2020).

Se recomienda de manera previa al procedimiento el uso de colutorio de clorhexidina gluconato $0,12 \%+$ Cloruro de Cetilpiridinio 0,05\%, antiséptico ampliamente aceptado que reduce la carga bacteriana de manera sustantiva disminuyendo complicaciones infecciosas pre y postoperatorias (Nicolosi et al., 2014; Cosola et al., 2019).

De acuerdo al contexto actual y como prevención para SARS-CoV-2, se podría utilizar peróxido de hidrógeno diluido al $1 \%$, efectivo como antiséptico puesto que el virus es vulnerable a la oxidación (Kampf et al., 2020; American Dental Association, 2020; Peng et al., 2020). También se ha descrito que la utilización de povidona yodada (PVP-I) en concentraciones de $0,23 \%$ a $7 \%$, demuestra una actividad virucida altamente efectiva contra una amplia gama de virus, incluidos varios coronavirus responsables de epidemias recientes, como SARS-CoV y MERS-CoV (Verbeek et al., 2020).

Se sugiere prescribir Amoxicilina $1000 \mathrm{mg}$ por vía oral, 2 veces al día durante 7 días (Romero-Ruiz et al., 2006), paracetamol $1000 \mathrm{mg}$ e ibuprofeno 400 $\mathrm{mg}, 3$ veces al día por un periodo mínimo de 5 días, comenzando con la terapia antibiótica 1 día antes de la cirugía y con la terapia analgesica y anti-inflamatoria 1 horas antes de esta, previniendo infección, inflamación y dolor quirúrgico (Beltrán et al., 2014; Gupta et al.). La dosis puede ser ajustada dependiendo del paciente, en caso de alergia se deben considerar alternativas farmacológicas de espectro y efecto similares. Es importante la utilización de anestesia troncular más complemento infiltrativo en primera instancia, evitando posibles refuerzos anestésicos durante el procedimiento sea este realizado con o sin sedación (Zhao \& Gao, 2020; Ellis et al., 2020; GonzálezQuintanilla \& Santos-López, 2020). Lidocaína al 2 \%, 
GONZÁLEZ-QUINTANILLA D.; ZAMORANO, J. P.; MELLA, E.; PINTO, N.; BRISSO, J.; RODRÍGUEZ, N. \& CASA, H. M. Autotrasplante dental utilizando simulación virtual y un prototipo de modelo de impresión 3D. Int. J. Odontostomat., 15(1):271-277, 2021.

es considerada una buena alternativa, dado el amplio conocimiento sobre sus efectos locales y sistémicos (Shah et al., 2018),

\section{CONSIDERACIONES QUIRÚRGICAS}

El procedimiento se debe llevar a cabo en salas de procedimiento con presión negativa como pabellones propiciando la esterilidad del procedimiento (Zhao \& Gao). La hidratación previa de los clínicos, antes de posicionar los elementos de protección personal, se recomienda para disminuir las probabilidades de error durante el procedimiento quirúrgico (Ellis et al.).

La exodoncia del diente comprometido debe ser ejecutada según las técnicas indicadas para él, con una delicada sindesmotomia para preservar la integridad de tejidos gingivales, además, de evitar la elongación de tablas de manera excesiva evitando fracturas (Tang et al., 2017). De manera consecutiva, se deben realizar las osteotomías previamente planificadas y simuladas virtualmente, junto con probar la adaptabilidad de la réplica del diente donante impreso en 3D previamente esterilizada con radiación gamma o beta, en el alvéolo receptor (Gulín Arias; Oh et al., 2018), posibilitando una disminución de la dificultad quirúrgica, aumentando la tasa de éxito del autotrasplante, disminución de la resorción inflamatoria de la raíz (Jang et al., 2016; Kafourou et al.) y la persistencia de poblaciones celulares que facilitan la reparación y regeneración de tejidos funcionales (Arima et al.).

La exodoncia del diente donante se debe ejecutar de la manera más atraumática posible (Verweij et al., 2016), limitando el uso de elevadores para evitar dañar el área del ligamento periodontal (Reich et al., 2008). Una vez luxado, se realiza la avulsión y trasplante inmediato al alvéolo preparado previamente, no excediendo un tiempo máximo de 5 minutos (Kim et al., 2005). Se debe procurar no tocar la zona del ligamento periodontal e implantarlo en un alvéolo o lecho quirúrgico receptor sano, con espacio corono-apical y mesio-distal adecuado que puede estar disponible o facilitado por osteotomías, cubierto de sangre y en inoclusión (Gupta et al.). En caso que el diente deba permanecer en un medio extraoral por más de 5 minutos se debe depositar en un frasco con solución salina equilibrada de Hank que mantiene la viabilidad de las células del ligamento periodontal (Ustad et al., 2002). El diente trasplantado debe ser ferulizado de manera semirrígida con hilo de pescar o fibra de polietileno fabricada por entrecruzado tipo leno, con el propósito de otorgar estabilidad y homologar movimientos fisiológi- cos que podrían facilitar la reparación y/o regeneración del ligamento periodontal (Vinitzky Brener et al., 2016). El tejido gingival debe ser re posicionado idealmente mediante sutura nylon $4 / 0$, evitando depósito de alimento y placa bacteriana. Esto optimiza la reinserción y puede bloquear la invasión bacteriana en el coágulo sanguíneo entre el diente y la cavidad (Ustad et al.).

La utilización o depósito de plasma rico en plaquetas, fibrina y/o fibrina rica en plaquetas entre otros productos autólogos y/o farmacológicos de naturaleza proteica en el lecho quirúrgico receptor, podría facilitar la diferenciación de células madre precursoras de ligamento periodontal y cemento a partir de osteoblastos, contribuyendo a la regeneración de tejidos de soporte (Tsukiboshi et al.; Melville et al., 2019).

\section{CONSIDERACIONES POSTQUIRÚRGICAS}

El paciente debe sostener una dieta blanda e higiene oral meticulosa. Se recomienda el uso de colutorio de clorhexidina gluconato $0,12 \%+$ Cloruro de Cetilpiridinio $0,05 \%$ por una semana para reducir la carga bacteria y promover la cicatrización o reparación tisular libre de infección (Ustad et al.).

Se debe considerar continuar con la medicación antibiótica, analgésica y anti-inflamatoria previamente prescrita de acuerdo a la condiciones sistémicas del paciente (Amoxicilina $1000 \mathrm{mg}$ por vía oral, 2 veces al día durante 7 días u otro antibiótico de similar espectro en caso de alergia, paracetamol $1000 \mathrm{mg} \mathrm{e}$ ibuprofeno $400 \mathrm{mg}, 3$ veces al día por un periodo mínimo de 5 días, asegurando cobertura durante el peak inflamatorio presente entre las 48 a 72 horas después del procedimiento) (Romero-Ruiz et al.; Beltrán et al.). En todos los casos, la prescripción y dosis es ajustable de acuerdo a las necesidades del paciente y complejidad del procedimiento.

Inicialmente se debe planificar un serie de controles, partiendo por una cita a las 24 o 48 horas, 1 semana, 2 semanas, 1 mes y los controles sucesivos hasta un periodo total de 5 años, planificados de acuerdo al riesgo del paciente con el propósito de favorecer un adecuado grado de funcionalidad. Es recomendable acompañar cada control con una radiografía periapical del diente en cuestión e instrucción de higiene (Kafourou et al.).

Se deben retirar las suturas 7 días después de la cirugía y la férula después de 2 semanas si el diente está adaptado a su alvéolo (Oh et al., 2018). En 
ambos casos, si no se cumplen los grados de adaptabilidad y/o funcionalidad, los autores de esta revisión recomiendan mantener las suturas y/o férula por una semana más e ir evaluando su mantención o retiro.

Si el paciente muestra una curación sin incidentes y se observa desarrollo radicular, facilitando el cierre del ápice de la raíz del diente trasplantado en las radiografías de seguimiento y sostiene normalmente el contacto oclusal con sus antagonistas después de 8 meses, el tratamiento se podría catalogar como exitoso (Oh et al., 2018).

Si existiera una complicación endodóntica, un especialista debe evaluar el tratamiento a indicar, sea este: la endodoncia convencional, reparación endodóntica guiada o tapón apical (Diogenes et al., 2013; Pace et al., 2014). La mantención del diente en la arcada aun cuando en el futuro signifique la anquilosis de este, también podría ser considerada como éxito clínico (Almpani et al., 2015).

\section{CONCLUSIONES}

La técnica de autotrasplante es una opción terapéutica actual ante la pérdida o ausencia de dientes por traumatismos, caries, agenesias, exfoliación temprana, iatrogenias, u otros factores. El trasplante de un diente desde su posición original para implantarlo en un solo acto quirúrgico en una zona edéntula, puede ser planificado y simulado virtualmente permitiendo optimizar posibles osteotomías y una implantación del diente donante de manera fácil, segura y predecible en tiempos quirúrgicos mínimos, favoreciendo la disminución de la complejidad quirúrgica, además de la reparación y regeneración de tejidos pulpares y periodontales. Existen indicaciones específicas para el uso de esta tecnología y no debe ser trivializada y empleada en casos de planificación y ejecución sencilla, dada su complejidad y costos asociados que se pueden transmitir a los pacientes.

GONZÁLEZ-QUINTANILLA D.; ZAMORANO, J. P.; MELLA, E.; PINTO, N.; BRISSO, J.; RODRÍGUEZ, N. \& CASA, H. M. Dental autotransplantation using virtual simulation and a prototype 3D printing model. Int. J. Odontostomat., 15(1):271277, 2021.

ABSTRACT: The dental autotransplantation technique was devised as a therapeutic option for the loss or absence of teeth due to trauma, caries, agenesis, early exfoliation, iatrogenesis, or other factors. It consists of extracting a tooth from its original position to implant it in a single surgical act in an edentulous area, be it a postextraction socket or a surgically made socket. A successful result requires meticulous selection of candidates, for this, the absence of pathologies and local or systemic conditions that hinder adequate wound healing and tissue repair must be considered. New tools to facilitate clinical success,such as planning and virtual simulation, together with the printing of prototypes in three dimensions (3D), would allow possible osteotomies and an implantation of the donor tooth in an easy way and in minimal surgical times, favoring the decrease of surgical complexity, in addition to the repair and regeneration of pulp and periodontal tissues. The objective of this article is to gather a series of considerations to optimize the dental autotransplantation technique.

KEY WORDS: dental autotransplantation, virtual simulation, 3D printing.

\section{REFERENCIAS BIBLIOGRÁFICAS}

Ahmed Asif, J.; Yusuf Noorani, T. \& Khursheed Alam, M. Tooth Autotransplantation: An Alternative Treatment. Bull. Tokyo Dent. Coll., 58(1):41-8, 2017.

Al Halabi, M.; Hussein, I.; Salami, A.; Awad, R.; Alderei, N.; Wahab, A. \& Kowash, M. A study protocol of a single-center investigatorblinded randomized parallel group study to investigate the effect of an acclimatization visit on children's behavior during inhalational sedation in a United Arab Emirates pediatric dentistry postgraduate setting as measured by the levels of salivary Alpha Amylase and Cortisol. Medicine (Baltimore), 98(35):e16978, 2019.

Almpani, K.; Papageorgiou, S. N. \& Papadopoulos, M. A. Autotransplantation of teeth in humans: a systematic review and meta-analysis. Clin. Oral Investig., 19(6):1157-79, 2015.

Alvarez, C.; Monasterio, G.; Cavalla, F.; Córdova, L. A.; Hernández, M.; Heymann, D.; Garlet, G. P.; Sorsa, T.; Pärnänen, P.; Lee, H. M.; et al. Osteoimmunology of oral and maxillofacial diseases: translational applications based on biological mechanisms. Front. Immunol., 10:1664, 2019.

American Dental Association. Coronavirus Frequently Asked. Chicago, American Dental Association, 2020. Disponible en: https://success.ada.org/en/practice-management/patients/ coronavirusfrequently-asked-questions

Andreasen, J. O.; Paulsen, H. U.; Yu, Z.; Bayer, T. \& Schwartz, O. A long-term study of 370 autotransplanted premolars. Part II. Tooth survival and pulp healing subsequent to transplantation. Eur. J. Orthod., 12(1):14-24, 1990.

Aparicio, P.; Basili, A. \& Castellón, L. Autotrasplante dentario: revisión de literatura y casos clínicos. Rev. Odontol. Mex., 12(4):22430, 2008.

Arima, M.; Hasegawa, D.; Yoshida, S.; Mitarai, H.; Tomokiyo, A.; Hamano, S.; Sugii, H.; Wada, N. \& Maeda, H. R-spondin 2 promotes osteoblastic differentiation of immature human periodontal ligament cells through the $\mathrm{Wnt} / \mathrm{b}$-catenin signaling pathway. J. Periodontal Res., 54(2):143-53, 2019.

Barrientos, S.; Cardozo, L. A. \& Rojas, L. M. Autotrasplantes dentales: revisión sistemática de la literatura. Univ. Odontol., 31(66):133-43, 2012. 
Beltrán, S. M.; Barrera, C. R.; Díaz, J. R.; Jaramillo, R. L.; Larraín, T. C. \& Valenzuela, V. C. Progresión de la respuesta inflamatoria sistémica. Rev. Chil. Cir., 66(4):333-40, 2014.

Clokie, C. M.; Yau, D. M. \& Chano, L. Autogenous tooth transplantation: An alternative to dental implant placement? J. Can. Dent. Assoc., 67:92-6, 2014.

Cosola, S.; Giammarinaro, E.; Marconcini, S.; Lelli, M.; Lorenzi, C. \& Genovesi, A. M. Prevention of bacterial colonization on suture threads after oral surgery: comparison between propolis- and chlorhexidine-based formulae. J. Biol. Regul. Homeost. Agents, 33(4):1275-81, 2019.

Diogenes, A.; Henry, M. A.; Teixeira, F. B. \& Hargreaves, K. M. An update on clinical regenerative endodontics. Endod. Top., 28(1):223, 2013.

Ellis, R.; Hay-David, A. G. C. \& Brennan, P. A. Operating during the COVID-19 pandemic: How to reduce medical error. Br. J. Oral Maxillofac. Surg., 58(5):577-80, 2020.

Giannobile, W. V. \& Lang, N. P. Are dental implants a panacea or should we better strive to save teeth? J. Dent. Res., 95(1):5-6, 2016.

González-Quintanilla, D. \& Santos-López, M. Preventive measures and considerations for oral surgery practice during COVID-19. Int. J. Odontostomat., 14(3):338-41, 2020.

Gulín Arias, E. J. Esterilización de ácido poliláctico y ácido poliglicólico mediante la utilización de radiación gamma y radiación beta. Máster. Madrid, Universidad Complutense de Madrid, 2014.

Gupta, S.; Goel, M.; Sachdeva, G.; Sharma, B. \& Malhotra, D. Autotransplantation. J. Conserv. Dent., 18(6):500-3, 2015.

Hamilton, T.; Markiewicz, M. R.; Jarman, J. \& Bell, R. B. Dental outcomes in computer- assisted orthognathic surgery. J. Craniofac. Surg., 23:e223-6. 2012.

Herrera, H.; Leonardo, M. R.; Herrera, H.; Miralda, L. \& Bezerra da Silva, R. A. Intentional replantation of a mandibular molar: case report and 14-year follow-up. Oral Surg. Oral Med. Oral Pathol. Oral Radiol. Endod., 102(4):85-7, 2006.

Jang, J. H.; Lee, S. J. \& Kim, E. Autotransplantation of immature third molars using a computer-aided rapid prototyping model: a report of 4 cases. J. Endod., 39(11):1461-6, 2013.

Jang, Y.; Choi, Y. J.; Lee, S. J.; Roh, B. D.; Park, S. H. \& Kim, E. Prognostic factors for clinical outcomes in autotransplantation of teeth with complete root formation: survival analysis for up to 12 years. J. Endod., 42(2):198-205, 2016.

Kafourou, V.; Tong, H. J.; Day, P.; Houghton, N.; Spencer, R. J. \& Duggal, M. Outcomes and prognostic factors that influence the success of tooth autotransplantation in children and adolescents. Dent. Traumatol., 33(5):393-9, 2017

Kampf, G.; Todt, D.; Pfaender, S. \& Steinmann, E. Persistence of coronaviruses on inanimate surfaces and their inactivation with biocidal agents. J. Hosp. Infect., 104(3):246-51, 2020.

Kang, J. Y.; Chang, H. S.; Hwang, Y. C.; Hwang, I. N.; Oh, W. M. \& Lee, B. N. Autogenous tooth transplantation for replacing a lost tooth: case reports. Restor. Dent. Endod., 38 (1):48-51, 2013.

Kim, E.; Jung, J. Y.; Cha, I. H.; Kum, K. Y. \& Lee, S. J. Evaluation of the prognosis and causes of failure in 182 cases of autogenous tooth transplantation. Oral Surg. Oral Med. Oral Pathol. Oral Radiol. Endod., 100(1):112-9, 2005.

Kurz, M.; Attin, T. \& Mehl, A. Influence of material surface on the scanning error of a powder-free 3D measuring system. Clin. Oral Investig., 19(8):2035-43. 2015.

Lee, S. J.; Jung, I. Y.; Lee, C. Y.; Choi, S. Y. \& Kum, K. Y. Clinical application of computer-aided rapid prototyping for tooth transplantation. Dent. Traumatol., 17(3):114-9, 2001

Lee, S. Y.; Huang, G. W.; Shiung, J. N.; Huang, Y. H.; Jeng, J. H.; Kuo, T. F.; Yang, J. C \& Yang, W. C. V. Magnetic cryopreservation for dental pulp stem cells. Cells Tissues Organs, 196(1):23-33, 2012.
Li, T.; Chang, J.; Zhu, Y. \& Wu, C. 3D printing of bioinspired biomaterials for tissue regeneration. Adv. Healthc. Mater., 9(23):2000208, 2020.

Lin, W. L.; McCulloch, C. A. \& Cho, M. I. Differentiation of periodontal ligament fibroblasts into osteoblasts during socket healing after tooth extraction in the rat. Anat. Rec., 240(4):492-506, 1994.

Liu, Y.; Di, P.; Zhao, Y.; Hao, Q.; Tian, J. \& Cui, H. Accuracy of multiimplant impressions using 3D-printing custom trays and splinting versus conventional techniques for complete arches. Int. J. Oral Maxillofac. Implants, 34(4):1007-14, 2019.

Mejàre, B.; Wannfors, K. \& Jansson, L. A prospective study on transplantation of third molars with complete root formation. Oral Surg. Oral Med. Oral Pathol. Oral Radiol. Endod., 97(2):231-8, 2004.

Melville, J. C.; Mañón, V. A.; Blackburn, C. \& Young, S. Current methods of maxillofacial tissue engineering. Oral Maxillofac. Surg. Clin. North Am., 31(4):579-91, 2019.

Meng, L.; Hua, F. \& Bian, Z. Coronavirus disease 2019 (COVID19): emerging and future challenges for dental and oral medicine. J. Dent. Res., 99(5):481-7, 2020.

Nicolosi, L. N.; del Carmen Rubio, M.; Martinez, C. D.; González, N. N. \& Cruz, M. E. Effect of oral hygiene and $0.12 \%$ chlorhexidine gluconate oral rinse in preventing ventilator-associated pneumonia after cardiovascular surgery. Respir. Care, 59(4):5049, 2014.

Nimc `enko, T.; Omerca, G.; Varinauskas, V.; Bramanti, E.; Signorino, F. \& Cicciù, M. Tooth auto-transplantation as an alternative treatment option: a literature review. Dent. Res. J. (Isfahan), 10(1):1-6, 2013.

Oh, S.; Kim, S.; Lo, H. S.; Choi, J. Y.; Kim, H. J.; Ryu, G. J.; Kim, S. Y.; Choi, K. K.; Kim, D. S. \& Jang, J. H. Virtual simulation of autotransplantation using 3-dimensional printing prototyping model and computer-assisted design program. J. Endod., 44(12):1883-8, 2018.

Oh, Y. H.; Che, Z. M.; Hong, J. C.; Lee, E. J.; Lee, S. J. \& Kim, J. Cryopreservation of human teeth for future organization of a tooth bank--a preliminary study. Cryobiology, 51(3):322-9, 2005.

Pace, R.; Giuliani, V.; Nieri, M.; Di Nasso, L. \& Pagavino, G. Mineral trioxide aggregate as apical plug in teeth with necrotic pulp and immature apices: a 10-year case series. J. Endod., 40(8):12504, 2014.

Peng, X.; Xu, X.; Li, Y.; Cheng, L.; Zhou, X. \& Ren, B. Transmission routes of 2019-nCoV and controls in dental practice. Int. J. Oral Sci., 12(1):9, 2020.

Reich, P. P. Autogenous transplantation of maxillary and mandibular molars. J. Oral Maxillofac. Surg., 66(11):2314-7, 2008.

Romero-Ruiz, M. M.; Herrero-Climent, M.; Torres-Lagares, D. \& Gutierrez-Pérez, J. L. Protocolo de control del dolor y la inflamación postquirúrgica. Una aproximación racional. RCOE, 11(2):205-15, 2006.

Shah, J.; Votta-Velis, E. G. \& Borgeat, A. New local anesthetics. Best Pract. Res. Clin. Anaesthesiol., 32(2):179-85, 2018.

Skoglund, A.; Hasselgren, G. \& Tronstad, L. Oxidoreductase activity in the pulp of re- planted and autotransplanted teeth in young dogs. Oral Surg. Oral Med. Oral Pathol., 52(2):205-9, 1981.

Strbac, G. D.; Schnappauf, A.; Giannis, K.; Bertl, M. H.; Moritz, A. \& Ulm, C. Guided autotransplantation of teeth: a novel method using virtually planned 3-dimensional templates. J. Endod., 42(12):1844-50, 2016.

Sugai, T.; Yoshizawa, M.; Kobayashi, T.; Ono, K.; Takagi, R.; Kitamura, N.; Okiji, T. \& Saito, C. Clinical study on prognostic factors for autotransplantation of teeth with complete root formation. Int. J. Oral Maxillofac. Surg., 39(12):1193-203, 2010.

Tang, H.; Shen, Z.; Hou, M. \& Wu, L. Autotransplantation of mature and immature third molars in 23 Chinese patients: a clinical and radiological follow-up study. BMC Oral Health, 17(1):163, 2017. 
GONZÁLEZ-QUINTANILLA D.; ZAMORANO, J. P.; MELLA, E.; PINTO, N.; BRISSO, J.; RODRÍGUEZ, N. \& CASA, H. M. Autotrasplante dental utilizando simulación virtual y un prototipo de modelo de impresión 3D. Int. J. Odontostomat., 15(1):271-277, 2021.

Tsukiboshi, M. Autotransplantation of teeth: requirements for predictable success. Dent. Traumatol., 18(4):157-80, 2002.

Ustad, F.; Ali, F. M.; Kota, Z. \& Mustafa, A. Autotransplantation of teeth: A review. Am. J. Med. Dent. Sci., 1:25-30, 2002.

Verbeek, J. H.; Rajamaki, B.; ljaz, S.; Sauni, R.; Toomey, E.; Blackwood, B.; Tikka, C.; Ruotsalainen, J. H. \& Balci, F. S. K. Personal protective equipment for preventing highly infectious diseases due to exposure to contaminated body fluids in healthcare staff. Cochrane Database Syst. Rev., 4(4):CD011621, 2020.

Verweij, J. P.; Jongkees, F. A.; Anssari Moin, D.; Wismeijer, D. \& van Merkesteyn, J. P. R. Autotransplantation of teeth using computeraided rapid prototyping of a three-dimensional replica of the donor tooth: a systematic literature review. Int. J. Oral Maxillofac. Surg., 46(11):1466-74, 2017.

Verweij, J. P.; Moin, D. A.; Mensink, G.; Nijkamp, P.; Wismeijer, D. \& van Merkesteyn, J. P. R. Autotransplantation of premolars with a 3-dimensional printed titanium replica of the donor tooth functioning as a surgical guide: proof of concept. J. Oral Maxillofac. Surg., 74(6):1114-9, 2016.

Vinitzky Brener, I.; Weihmann Sánchez, E. P.; Aguilar Rojas, A. M. \& Peña Anaya, E. et al. Autotrasplante dental. Revisión de la literatura y presentación de dos casos. Rev. ADM, 73(4):212-7, 2016.

Zhao, Z. \& Gao, D. Precaution of 2019 novel coronavirus infection in department of oral and maxillofacial surgery. Br. J. Oral Maxillofac. Surg., 58(3):250-3, 2020.
Dirección para correspondencia:

David González Quintanilla

Facultad de Ciencias de la Salud

Universidad Autónoma de Chile

Santiago

CHILE

E-mail: davidgonzalez.odonto@gmail.com 